Au-delà du développement durable, une éducation à la compétence éthique dans la diversité et la complexité des cultures

\title{
Milagros Chávez
}

\section{CpenEdition}

Journals

Édition électronique

URL : https://journals.openedition.org/ere/5501

DOI : $10.4000 /$ ere.5501

ISSN : 2561-2271

Éditeur

Centr'ERE

\section{Référence électronique}

Milagros Chávez, «Au-delà du développement durable, une éducation à la compétence éthique dans la diversité et la complexité des cultures », Éducation relative à l'environnement [En ligne], Volume 4 | 2003, mis en ligne le 14 septembre 2003, consulté le 21 septembre 2021. URL : http:// journals.openedition.org/ere/5501; DOI : https://doi.org/10.4000/ere.5501

Ce document a été généré automatiquement le 21 septembre 2021.

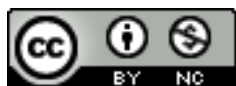

La revue Éducation relative à l'environnement est mise à disposition selon les termes de la Licence Creative Commons Attribution - Pas d'Utilisation Commerciale 4.0 International. 


\title{
Au-delà du développement durable, une éducation à la compétence éthique dans la diversité et la complexité des cultures
}

\author{
Milagros Chavez
}

1 Nous avons souvent entendu parler de la crise environnementale qui afflige notre planète et de l'urgence de prendre les mesures nécessaires pour éviter la catastrophe. Certains auteurs, tels que Bowers (1993), Aramburu (2000), Jonas (2000), Monod (2000) et Moscovici (2002) sont d'accord pour affirmer que cette crise trouve son origine dans une certaine compréhension du monde, des choses et de l'être humain.

2 Pour Aramburu (2000) et Monod (2000), cette manière de comprendre le monde a pour origine la conception mythico-religieuse occidentale. Celle-ci donne à l'être humain une position de prédominance, de propriété et de contrôle sur la création (la nature). Selon cette conception, l'être humain, en tant que suprême résultat de la création - ou de l'évolution dans une version plus moderne -, aurait le droit (et le mandat) de soumettre la nature à ses besoins et ses desseins.

3 Cette conception de la nature fait partie de l'ensemble des mythes et des croyances de ce qu'on peut appeler la « culture occidentale moderne » (Perrot et coll., 1992). Si nous suivons la définition de la culture donnée par Clifford (cité par Bowers, 1993, p. 21), la culture est :

un ensemble de patrons partagés par une communauté, désignant une représentation de multiples éléments provenant de la religion (de la spiritualité et de la transcendance), de la philosophie, de l'esthétique, de la science, de l'idéologie, de la politique, de l'économie, de la relation à l'environnement, etc.

Les pays qui ont vécu le processus de la révolution industrielle et technologique des 19e et 20e siècles (la plupart appartenant à l'hémisphère nord et occidental de la planète) partagent en effet un ensemble de patrons et de représentations qui guident les conduites et les actions de leurs habitants (Perrot et coll., 1992 ; Bowers, 1993). 
Un exemple de ces patrons et représentations est le modèle de développement centré sur le progrès scientifique et technologique, sur la croissance économique et sur la consommation de biens. Les valeurs ${ }^{1}$ à la base de cette représentation se centrent sur une recherche continue de sécurité et de confort par l'individu (propriété privée, consommation des biens et services, etc.). Cette situation produit un système de relations interpersonnelles basé sur la concurrence et l'intérêt individuel. Ces mêmes valeurs individualistes sont projetées (reflétées) dans la relation avec l'environnement. On considère que ce dernier se trouve au service de l'être humain. Il est là pour être utilisé et exploité de manière à couvrir les nécessités et les luxes de l'humanité. Jusqu'à récemment, on croyait qu'il pouvait en être ainsi indéfiniment. Mais, en grand partie à cause de l'avancement de la science et de la technologie, nous avons eu le pouvoir de produire des changements sur l'environnement à une vitesse et dans une mesure telles que la nature n'a pas eu le temps de se restaurer. On sonne maintenant l'alarme à la crise environnementale. Mais il est important de comprendre que cette crise environnementale est aussi, simultanément et de manière très imbriquée, une crise sociale. Les valeurs individualistes de la culture occidentale moderne nous séparent des individus de notre propre espèce et du reste de la nature. Il s'agit d'une crise socioenvironnementale.

4 La question qui se pose est de savoir comment trouver une solution (voire des solutions) profonde(s) à cette crise, sans prétendre introduire des bouleversements radicaux aux patrons culturels des pays occidentaux et occidentalisés. Sur ce point, la Commission mondiale sur l'environnement et le développement (1998) dans son document Notre avenir à tous a proposé une idée apparemment conciliatrice entre les mouvements écologistes réformateurs, contestataires et les défenseurs du développement économique. Il s'agit de la possibilité de s'inscrire dans une logique de " développement durable » qui réponde aux besoins des populations d'aujourd'hui sans mettre en péril les possibilités de bien-être des futures générations. Cette proposition a été largement diffusée à l'occasion du sommet de Rio en 1992 dans le document «Action 21 ». Elle a aussi été l'objet d'une série d'engagements de la part de certains pays participants à ce sommet.

Bien entendu, les intentions explicites du concept de développement durable sont très louables et il faut reconnaître que celui-ci a permis de débloquer le dialogue entre les groupes écologistes, les décideurs politiques et le monde des affaires. Mais malheureusement, le concept en question, trop flou, est source de confusion. Il peut prendre des sens totalement différents et même contradictoires selon les interprétations qu'on lui donne (Sauvé, 2000). Tout en engageant le dialogue, on a ouvert la porte à la confusion. C'est ainsi qu'on utilise les mêmes termes de développement durable pour faire référence aux normes de contrôle environnemental ISO 14000 des pays industrialisés et aux pratiques ancestrales de peuples autochtones. En fait, on parle de cultures complètement distinctes qui ne partagent pas le même concept de développement, ni de durabilité.

6 Par ailleurs, il est possible de faire un lien entre le concept de développement (se trouvant à la base de l'idée de développement durable) et celui de progrès (tel qu'il est compris par le positivisme). Les origines de l'idée de progrès dans les pays occidentaux remontent au temps où le degré de confiance dans la raison humaine (René Descartes) et dans les sciences (Auguste Comte) permettaient de concevoir que les possibilités de l'être humain étaient illimitées. Les processus d'industrialisation, s'appuyant sur 
l'épanouissement des sciences et des technologies, n'ont fait que renforcer cette représentation. Pour sa part, le concept de développement est apparu dans la première partie du 20e siècle, lorsque les pays industrialisés se sont vus dans la nécessité (et,ou dans l'intérêt) d'exporter leurs systèmes de production et d'exploitation (ainsi que les croyances positivistes et scientistes que ceux-ci véhiculaient implicitement) dans d'autres régions de la planète. Ils ont alors défini leur mode de vie, comme le plus approprié, leur culture comme la plus civilisée, tandis que les autres modes de vie et de relation avec la nature, dans d'autres régions du monde, étaient considérés comme des modèles de production et d'exploitation peu productifs et peu efficaces, se trouvant en retard par rapport à ceux des pays industrialisés ${ }^{2}$.

7 Le développement (industriel et économique) des pays du Nord et de l'Occident, basé sur l'idée de progrès, est ainsi devenu le modèle de développement à suivre pour toutes les régions. Bien sûr, ce modèle s'accompagnait aussi d'une dimension sociale, de caractère positiviste. On s'attendait à ce que le développement scientifique, industriel et économique ait comme conséquence le bien-être du plus grand nombre de personnes possible dans toutes les régions. Mais la réalité indique que les projets de développement destinés (imposés?) aux pays «sous-développés » n'ont pas accompli cette attente. Au contraire, les pauvres sont devenus plus démunis et les riches plus puissants (Perrot et coll., 1992).

8 Par ailleurs, étant donné les limites mêmes de la nature et la vitesse du développement, le bien-être du plus grand nombre de personnes possible a commencé à être difficile à garantir. Les communautés de toutes les régions de la planète ont commencé à subir les conséquences de la surexploitation et de la pollution produites par le développement.

C'est à ce moment que le concept de développement durable fait son apparition. Avec lui, on évoque la possibilité de concevoir un développement scientifique, social, industriel et économique qui ait un impact minimal sur l'environnement. On construit le concept sur la base de trois piliers : l'économique, le social et l'environnemental. Cependant, tel que nous l'explique Sauvé (2000), dans la plupart des interprétations du développement durable, on continue à mettre l'accent sur le développement économique, tandis que l'environnement et la dimension sociale sont perçus comme des contraintes dont il faut tenir compte.

Bien entendu, il y a eu une avancée par rapport à l'idée de développement tout court, mais les valeurs à la base du projet n'ont pas changé en profondeur. Ainsi, la relation entre les humains et l'environnement est toujours centrée sur l'utilitaire. L'environnement est là pour être utilisé, pour répondre à nos « besoins ». La différence est que, dans le développement durable, cette utilisation doit être plus rationnelle. L'humain, d'exploiteur qu'il était dans l'idée de développement, devient le gestionnaire de l'environnement dans le développement durable (Sauvé, 2000). Mais, le bénéficiaire ultime reste l'être humain, et les valeurs de sécurité et de confort se trouvent toujours au centre de l'action.

11 Dans la sphère de l'éducation relative à l'environnement, et plus spécifiquement dans l'éducation à l'éthique de l'environnement, un des principaux problèmes du concept du développement durable est l'hégémonie gagnée par celui-ci. Le caractère unificateur et général du terme "développement durable» a incité certaines organisations internationales telles que l'UNESCO, l'OCDE, la Banque Mondiale, etc. à promouvoir l'éducation pour le développement durable en tant que centre et fondement de l'éducation relative à l'environnement et de l'éducation en général. 
12 Toutefois, en se centrant sur l'éducation au développement durable, l'éducation relative à l'environnement risque de perdre ses possibilités de critique profonde et subversives. En effet, comment peut-on questionner des valeurs fondamentales de la culture occidentale moderne tels que l'individualisme ou la surconsommation et en même temps défendre la nécessité de développer les marchés économiques ? Comment peut-on critiquer les avancées parfois délirantes (et irrationnelles) de la science et de la technologie alors qu'on fait parallèlement la promotion de son progrès comme base du développement?

13 L'éducation relative à l'environnement perçue comme une dimension fondamentale de l'éducation de la personne, c'est-à-dire de l'être éduqué (Legendre, 1995), doit conserver son caractère critique et subversif. C'est une éducation qui doit encourager l'analyse des diverses éthiques de l'environnement qui ont existé et qui existent (Des Jardins, 1995), de la multitude de valeurs se rattachant à l'environnement et de la diversité de styles de rapports à ce dernier. Il doit s'agir d'une éducation qui produise une ouverture profonde des mentalités afin de pouvoir regarder au-delà, dans une perspective critique, les patrons et les mythes de la culture occidentale moderne tel que ceux du développement durable.

Mais comment produire une telle éducation? Il n'est guère facile de donner une réponse à cette question. Bien sûr, la stimulation de la pensée critique fait partie de la réponse, de la même manière que l'analyse et la clarification des valeurs.

15 Le centre d'attention d'une telle éducation doit à mon sens être le développement de la compétence éthique. On peut comprendre celle-ci comme la capacité (continuellement en évolution) d'assumer une position responsable, de prendre des décisions et d'entreprendre des actions, sur la base d'une réflexion critique et contextuelle de nos propres valeurs et représentations ainsi que de celles des autres. Cette compétence implique la possibilité de prendre une distance critique par rapport aux valeurs qui nous mobilisent et qui sont très imbriquées dans notre culture.

16 Pour construire cette compétence éthique, un premier pas pourrait être d'analyser la représentation de l'environnement et du rapport à celui-ci dans des cultures différentes des cultures occidentales (autochtones de toute l'Amérique, d'Afrique, du Moyen-Orient, d'Asie, etc.). Ces relations à l'environnement reflètent différents systèmes de valeurs qui peuvent servir pour mettre en perspective nos propres valeurs.

17 Quelle est la signification des autres êtres vivants se trouvant dans l'environnement? À qui appartient l'environnement? Quel est le rôle de l'humain dans l'environnement? Quelle est la dépendance de l'humain par rapport à l'environnement? Voilà quelques questions qui peuvent servir à lancer une réflexion et une analyse du rapport à l'environnement tel qu'il est perçu dans d'autres cultures. À partir de cette première analyse, nous pourrons continuer en tentant de clarifier nos propres valeurs en fonction des mêmes questions. Cette fois, nous nous poserons nous-mêmes ces questions, de manière personnelle et aussi collective. Il s'agit de produire une ouverture de notre pensée vers des horizons nouveaux, un acte créatif dans la recherche d'une prise de position sur la base d'un univers plus global que celui que nous impose notre propre culture. Il s'agit d'une éducation valorisant la multiculturalité de notre planète et l'importance de l'authenticité de nos choix comme personnes et comme collectivité. 


\section{BIBLIOGRAPHIE}

Aramburu, F. (2000). Medio ambiente y educación. Madrid : Síntesis, Collection Didáctica de las Ciencias Sociales.

Bowers, C.A. (1993). Education, cultural myths and the ecological crisis : Toward deep changes. New York : State University of New York Press.

Commission mondiale sur l'environnement et le développement. (1998). Notre avenir à tous (Rapport Brundtland). Montréal : Éditions du Fleuve.

Desjardins, J.R. (1995). Éthique de l'environnement : Une introduction à la philosophie de l'environnement. Sainte-Foy : Presse de l'Université du Québec.

Jonas, H. (2000). Une éthique pour la nature. Paris : Éditions Desclée de Brouwer.

Legendre, R. (1995). Entre l'angoisse et le rêve. Montréal : Guérin-Eska.

Monod, T. (2000). Et si l'aventure humaine devait échouer. Paris : Grasset et Fasquelle.

Moscovici, S. (2002). De la nature : pour penser l'écologie. Paris : Métaillié.

Perrot, M.-D., Rist, G. et Sabelli, F. (1992). La mythologie programmée : L'économie des croyances dans la société moderne. Paris : Presses universitaires de France.

Sauvé, L. (2000). L'éducation relative à l'environnement - Entre modernité et postmodernité : Les propositions du développement durable et de l'avenir viable. Dans A. Jarnet, B. Jickling,

L. Sauvé et P. Clarkin (dir.), A colloquium on the future of environmental education in a postmodern world. Whitehorse : Canadian Journal of Environmental Education, p. 57-70.

\section{NOTES}

1. Dans cette analyse, le mot «valeurs » exprime les principes, conscients ou inconscients, qui guident l'action de la personne et des groupes.

2. Selon Perrot et coll. (1992, p. 188), « le terme développement a été officialisé pour la première fois dans l'article 22 du pacte de la Société des Nations (1919) qui déclare notamment que « le bien-être et le développement » de colonies et territoires placés sous mandat forment une mission sacrée de civilisation »"

\section{INDEX}

Keywords : environmental education, naturalism, consumption, culture, relations to the environment, sustainable development

Mots-clés : éducation relative à l'environnement, naturalisme, consommation, culture, rapports à l'environnement, développement durable 


\section{AUTEUR}

\section{MILAGROS CHAVEZ}

Milagros Chavez est professeure et chercheure en éducation relative aux sciences et à l'environnement à l'Universidad de los Andes de Mérida, Venezuela, depuis 1992. Actuellement inscrite au doctorat en éducation de l'Université du Québec à Montréal, sa thèse a pour titre : L'éducation relative à l'environnement comme dimension transversale de l'éducation scientifique : vers un modèle éducationnel, sous la direction de Lucie Sauvé et la codirection de Gilles Thibert. 